\title{
Hospitalization Duration Following Uncomplicated Cesarean Delivery: Predictors, Facility Variation, and Outcomes
}

\author{
Jerome J. Federspiel, MD, PhD ${ }^{1,2}$ Sunitha C. Suresh, MD ${ }^{1,3} \quad$ Kristin C. Darwin, MD ${ }^{1}$
}

Linda M. Szymanski, MD, $\mathrm{PhD}^{1,4}$

${ }^{1}$ Department of Gynecology and Obstetrics, Johns Hopkins University School of Medicine, Baltimore, Maryland

2 Department of Obstetrics and Gynecology, Duke University School of Medicine, Durham, North Carolina

${ }^{3}$ Department of Obstetrics and Gynecology, University of Chicago, Chicago, Illinois

${ }^{4}$ Department of Obstetrics and Gynecology, Mayo Clinic, Rochester, Minnesota

Address for correspondence Jerome J. Federspiel, MD, PhD, Department of Gynecology and Obstetrics, Johns Hopkins University, 600 North Wolfe Street, Phipps 218, Baltimore, MD 21287 (e-mail: jfederspiel@gmail.com).

Am J Perinatol Rep 2020;10:e187-e197.

\begin{abstract}
Keywords

- cesarean section/economics

- cesarean section/utilization

- pregnancy

- cesarean delivery

- length of stay

- readmission

- facility variation

Objectives This study was designed to: (1) characterize stay duration following cesarean delivery, (2) ascertain whether facility variation exists, and (3) determine whether shorter stays are associated with rates of readmission or costs.

Study Design The 2017 Nationwide Readmissions Database was used to identify uncomplicated cesarean deliveries. Hierarchical logistic regression was used to assess for facility variation in percentage of patients discharged within 2 days. Similar models were used to assess for associations between probability of readmission within 30 days and facility-level rates of discharge within 2 days.

Results In total, 456,312 patients from 1,535 hospitals were included. The median facility discharged $46.8 \%$ of patients within 2 days, with the 25 th percentile of hospitals $23.7 \%$ and the 75 th percentile $71.2 \%$. In adjusted regression, there was significant facility heterogeneity $(p<0.0001)$. The overall readmission rate was $1.7 \%$, and proportion of patients discharged within 2 days of cesarean delivery was not associated with readmission probability (adjusted relative risk: 1.02, confidence interval: 0.90-1.16), but was associated with lower inpatient costs (adjusted incremental cost: $\$ 111$, confidence interval: -181 to -41 ).

Conclusion Unexplained facility variation in percentage of patients discharged within 2 days of cesarean delivery was not associated with differences in readmissions.
\end{abstract}

The duration of hospitalization following many types of surgery continues to decline in the United States. ${ }^{1}$ Obstetrics and gynecology is no exception to this trend; total laparoscopic hysterectomy is now commonly a same-day, outpatient procedure. $^{2}$ However, studies of length of stay following childbirth in the United States have been colored by concern regarding inappropriately early obstetric discharge. Between the 1970s and middle 1990s, there was a dramatic reduction in length of stays following both vaginal and cesarean deliveries. ${ }^{3}$ This trend captured considerable popular attention due to concerns that patients were discharged from the hospital prematurely, with these trends described as creating received

December 8, 2019

accepted

March 5, 2020
DOI https://doi.org/

10.1055/s-0040-1709681. ISSN 2157-6998.
Copyright $\odot 2020$ by Thieme Medical Publishers, Inc., 333 Seventh Avenue, New York, NY 10001, USA. Tel: +1(212) 760-0888.
License terms

(요 (1) $\Theta$ 
"drive through deliveries." 4 Based on these concerns, states passed legislation mandating entitlement to a fixed number of hospital days following vaginal and cesarean deliveries. This culminated with federal legislation via passage of the Newborns' and Mothers' Health Protection Act (NMHPA) of 1996, which created an entitlement to a 96-hour hospitalization after cesarean delivery for patients covered by many types of private insurance plans. While mothers are entitled to this duration of hospitalization, patients may be discharged earlier based on assessment of their attending physician.

Since passage of the NMHPA more than 20 years ago, there has been continued improvements in the evidence-based informing performance of cesarean delivery and anesthetic practice. ${ }^{5}$ Furthermore, American health care is known to have substantial variation in practice patterns based on the type of facilities in which patients receive treatment. ${ }^{6}$ Whether this variation and its potential impact on outcome exists in postcesarean discharge duration is unknown. Thus, the objectives of this study were to (1) characterize contemporary duration of stay following cesarean delivery in the United States, (2) ascertain whether facility variation exists in duration of stay, and (3) determine whether shorter durations of stay (of 2 days or fewer) are associated with differences in rates of hospital maternal readmission or costs.

\section{Materials and Methods}

We used data from the 2017 Nationwide Readmissions Database (NRD), Healthcare Cost and Utilization Project, Agency for Healthcare Research and Quality. ${ }^{7}$ The 2017 NRD is an allpayor administrative dataset containing most acute care, short stay hospitalizations for 28 states, comprising $60.0 \%$ of the U.S. population and $58.2 \%$ of all hospitalizations. The data in each record includes demographic information about the patient, including age, gender, diagnosis and procedure codes, information about the treating hospital including location and academic affiliation, and outcome information including length of stay, inpatient charges, and discharge disposition. Record linkage allows identification of subsequent hospitalizations across the year for the same patient (within the same state). Cost-tocharge ratio files are provided with the data to enable conversion from hospital charges to estimated hospital cost.

Patients who underwent a low transverse cesarean delivery were included based on the use of International Classifications of Diseases, 10th Edition, Clinical Modification (ICD-10-CM) procedure code 10D00Z1. The NRD includes variables indicating the number of days from date of admission to the date each procedure was performed, and as well as total length of stay. The difference between these two variables was used to calculate the postcesarean length of stay (i.e., the length of stay presented here is the time subsequent to cesarean delivery). Only patients whose discharge destination was to home (with or without home care) were included. This study was intended to focus on low-risk patients. We identified both the presence of significant maternal morbidity during the patients' delivery hospitalization, as well as comorbid conditions, using a previously validated algorithm, which we updated to ICD-10-CM diagnostic codes. ${ }^{8}$ All patients who had evidence of significant maternal morbidity were excluded, as were patients who had a comorbid condition that, in our clinical opinion, might necessitate longer inpatient postoperative care (specifically, pulmonary hypertension, sickle cell disease, preeclampsia, chronic kidney disease, ischemic heart disease, congenital or valvular cardiac disease, cystic fibrosis, or morbidly adherent placenta). To further limit the cohort to patients who might be considered reasonable for early discharge, patients whose delivery was complicated by preterm birth, fetal demise, maternal cardiovascular disease, uterine rupture, and delayed twin birth were removed as identified in published quality indicators for primary cesarean delivery updated to ICD-10-CM criteria by us, ${ }^{9}$ and we additionally excluded chorioamnionitis $(041.1 \mathrm{x})$. Due to the need for a 30-day follow-up period to calculate readmission rates, patients discharged in December 2017 were excluded. Patients who had multiple delivery records in the same year or for whom the date of the cesarean delivery procedure was unavailable were omitted, since it would not be possible to calculate the postdelivery length of stay without this information. As facility variation was a key focus of the analysis, observations from facilities in which fewer than 10 patients met study criteria were excluded.

The data were summarized using mean and median for continuous data and percentages for categorical data. Comorbid conditions and indications for cesarean delivery were identified using previously described criteria from the discharge diagnosis codes, updated to ICD-10-CM. ${ }^{8,10}$ Weights were incorporated to reflect the sampling strategy underlying the NRD. To assess for predictors of shorter hospitalizations (defined as a postcesarean length of stay of 2 days or less), a hierarchical logistic regression was then used to model hospital length of stay less than or equal to 2 days as a function of patient and hospital characteristics, incorporating variation at the hospital level using random effects. The variation in lengths of stay following cesarean delivery at the facility level were then calculated, based on both percentage of patients discharged within 2 days of delivery and by mean length of stay. To assess whether variations in facility-level length of stay following cesarean delivery were associated with differences in an individual's probability of readmission and inpatient costs, regression models (logistic for readmission and gamma log link for costs) were constructed, predicting outcome as a function of the patient's individual characteristics, hospital characteristics, and the facility rate of discharge within 2 days of delivery, with standard errors clustered by facility. To improve interpretability, the results were transformed from their native odds ratio scale for logistic regression to relative risks and absolute risk differences, and from the native multiplicative effect for the gamma log link regression to an incremental effect. As each individual's length of stay is likely confounded with their outcome, when calculating the facility length of stay metrics, the metric was recalculated for each individual to exclude that individual's length of stay. Alternative formulations of these models, including specifying the facility rate using quadratic, categorical (quartiles), and a restricted cubic spline using three quartile-based knots were performed to assess robustness to the choice of model specification, and an alternative measure of 
facility length of stay (mean length of stay) was also modeled to assess robustness to choice of measure for the readmission outcome.

A two-sided $\alpha$ value of 0.05 was specified as statistically significant. Missing data elements were minimal ( $<1 \%$ for all variables) and were addressed with imputing based on modal value. The data were analyzed in Stata Statistical Software, Version 16.1 (Statacorp, College Station, TX). Given this study is a retrospective analysis using an existing limited dataset, the Johns Hopkins University School of Medicine Institutional Review Board determined it to be exempted from review.

\section{Results}

A total of 456,312 patients who delivered at 1,535 facilities met inclusion criteria ( $\mathbf{- F i g . 1}$ ). When weighted to produce estimates of the national population, rather than only the sample included in the NRD dataset, this sample equates to 848,556 patients. Of these patients, the median length of stay was 3 days, and the mean length of stay was 2.7 days. Very few $(1.8 \%)$ patients stayed less than 2 days following cesarean delivery, or more than 4 days $(1.2 \%)$; most patients stayed 2 days (39.1\%), 3 days (46.4\%), or 4 days (11.5\%). Mean age in the cohort was 29.9 years, the majority $(54.0 \%)$ was insured by private insurance, and most (52\%) had a history of prior cesarean delivery ( - Table $\mathbf{1}$ ). The overall maternal readmission rate was $1.7 \%$, and total inpatient costs were estimated at $\$ 6,800$ per admission.

When patients were stratified by length of stay following cesarean delivery (-Table $\mathbf{1}$ ), shorter hospital stays were associated with younger age (mean age 29.3 for 2-day stays and 31.1 for 4-day stays), insurance coverage by Medicaid rather than private insurance, and lower rates of most comorbid conditions. Patients with shorter stays were also more likely to have had a prior cesarean section $(60.1 \%$ of those hospitalized for 2 days and $40.5 \%$ of those hospitalized for 4 days). Shorter hospital stays were more common at government-owned or private for-profit hospitals than private nonprofit hospitals and were also more common at nonteaching hospitals in both metropolitan and nonmetropolitan settings. The ZIP code median income of patients hospitalized for 2 days after cesarean section was lower than patients hospitalized for 4 days. In unadjusted analyses, shorter hospital stays were associated with lower rates of readmission, lower total hospital charges, and lower total hospital costs. After regression modeling for the other measured possible predictors of 2-day discharge, several predictors were associated with length of stay following cesarean delivery ( - Table 2 ). Predictors associated with greater probability of discharge within 2 days postsurgery included other insurance status (compared with privately insured patients), receipt of care in a nonmetropolitan hospital (compared with a metropolitan, nonteaching hospital), prior cesarean delivery, and tobacco use disorder. Factors associated with lower probability of 2-day or earlier discharge included increasing age, coverage with Medicare or Medicaid, care in a nonprofit hospital (vs. a government hospital), receipt of care in a metropolitan, teaching hospital (vs. a metropolitan, nonteaching hospital), increasing ZIP code-level median household income (vs. decreasing in the unadjusted results), most comorbid conditions, and most indications for cesarean delivery other than history of prior cesarean delivery.

There was significant facility variation in the duration of stay following cesarean delivery (- Table 1, - Fig. 2). - Fig. 2A depicts the number of hospitals (as a percentage) that discharged patients on postoperative day 2 or sooner, when expressed as a percentage of their total postcesarean discharges. The median facility discharged $47.2 \%$ of patients within 2 days of delivery, with the 25 th percentile 23.8 and the 75 th percentile $71.3 \%$. - Fig. 2B shows the distribution of hospitals according to average length of stay in days. The overall mean length of stay was 2.6 days, with 25 th percentile 2.3 days and 75 th percentile 2.9 days.

When facilities were stratified into quartiles by percentage of patients discharged on day 2 or sooner (-Table 3 ), patients treated at facilities with the highest rate were younger, more likely to be covered by Medicaid, lived in ZIP codes with lower median household incomes, and were more likely to have had a prior cesarean section. The hospitals were more likely to be government, nonteaching or nonmetropolitan hospitals, and to have had fewer cases included in the cohort. Rates of placenta previa, drug abuse, and asthma were lower in these facilities with higher rates of 2-day discharge, whereas rates of tobacco use were higher.

In unadjusted analyses, facility rates of 2-day or earlier discharge were associated with no statistically significant difference in 30-day, all-cause readmission (relative risk: 1.03, 95\% confidence interval: 0.91-1.16) (-Fig. 3). After regression adjustment, this difference remained not statistically significant (relative risk: $1.02,95 \%$ confidence interval: $0.90-1.16)$. This corresponds to an absolute risk difference of $0.04 \%$ (95\% confidence interval: -0.17 to $0.24 \%$ ). These findings were robust to other specifications of the facility variation (changing functional form to use squared term, quartiles, or restricted cubic spline), specification using mean length of stay rather than percentage discharged in 2 days or less, and for readmission, use of a 42-day time frame rather than a 30-day time frame. The facility rate of discharge was associated with lower inpatient costs in both unadjusted (percentage reduction: 17\%, 95\% confidence interval: 8-25\%) and adjusted models (percentage reduction: 15\%; 95\% confidence interval: 6-23\%). This corresponds to an average incremental cost difference of $\$ 111$ ( $95 \%$ confidence interval: -181 to -41$)$.

\section{Discussion}

In this retrospective cohort study in a large population of low-risk patients undergoing cesarean deliveries uncomplicated by either high-risk comorbid conditions or incidence of maternal morbidity, virtually all patients were discharged home on postoperative day 2,3 , or 4 . There was broad facility variation in the percentage of patients discharged home on day 2 or earlier, which could not be explained by patient or hospital characteristics. After regression adjustment, 


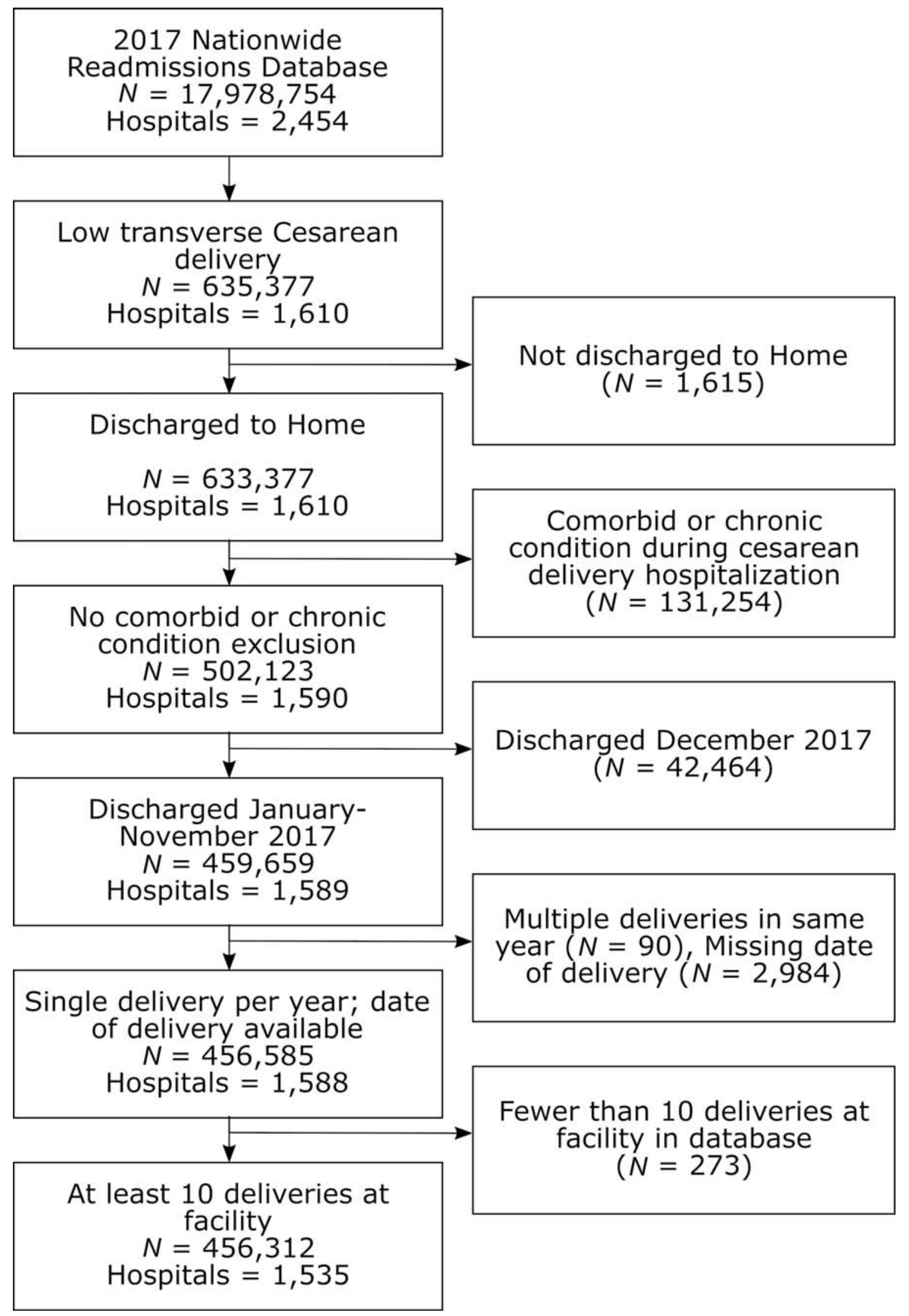

Fig. 1 Derivation of study sample. 
Table 1 Baseline patient and facility characteristics, stratified by length of stay

\begin{tabular}{|c|c|c|c|c|c|c|c|}
\hline \multirow[b]{3}{*}{$\begin{array}{l}\text { Mean (standard deviation) } \\
\text { or \% }\end{array}$} & \multicolumn{7}{|c|}{ Length of stay following cesarean delivery (d) } \\
\hline & \multirow{2}{*}{$\begin{array}{l}\text { Overall } \\
(N=456,312) \\
\text { (weighted } \\
N=848,556)\end{array}$} & \multirow{2}{*}{$\begin{array}{l}<2 \mathrm{~d} \\
(N=8,276) \\
\text { (weighted } \\
N=15,533)\end{array}$} & \multirow{2}{*}{$\begin{array}{l}2 \mathrm{~d} \\
(N=178,474) \\
\text { (weighted } \\
N=332,781)\end{array}$} & \multirow{2}{*}{$\begin{array}{l}\mathrm{d} \\
(N=211,605) \\
\text { (weighted } \\
N=394,795)\end{array}$} & \multirow{2}{*}{$\begin{array}{l}4 \mathrm{~d} \\
(N=52,693) \\
\text { (weighted } \\
N=96,012)\end{array}$} & \multirow{2}{*}{$\begin{array}{l}>4 \mathrm{~d} \\
(N=5,264) \\
\text { (weighted } \\
N=9,435)\end{array}$} & $p$-Value \\
\hline & & & & & & & \multirow[b]{2}{*}{$<0.001$} \\
\hline Age in $y$ at admission & $29.9(5.7)$ & $29.2(5.5)$ & $29.3(5.5)$ & $30.0(5.8)$ & $31.1(5.9)$ & $30.5(6.3)$ & \\
\hline \multicolumn{8}{|l|}{ Primary payer } \\
\hline Medicare & $7,953(0.9)$ & $93(0.6)$ & $2,551(0.8)$ & $3,731(0.9)$ & $1,331(1.4)$ & $247(2.6)$ & \multirow{6}{*}{$<0.001$} \\
\hline Medicaid & $349,491(41.2)$ & $6,731(43.3)$ & $142,285(42.8)$ & $162,534(41.2)$ & $33,849(35.3)$ & $4,093(43.4)$ & \\
\hline Private & $457,887(54.0)$ & $7,693(49.5)$ & $173,122(52.0)$ & $214,465(54.3)$ & $57,845(60.2)$ & $4,763(50.5)$ & \\
\hline Self-pay & $10,281(1.2)$ & $454(2.9)$ & $4,400(1.3)$ & $4,443(1.1)$ & $855(0.9)$ & $129(1.4)$ & \\
\hline No charge & $416(0.0)$ & $18(0.1)$ & $137(0.0)$ & $217(0.1)$ & $33(0.0)$ & $(0.1)^{\mathrm{a}}$ & \\
\hline Other & $22,529(2.7)$ & $544(3.5)$ & $10,286(3.1)$ & $9,405(2.4)$ & $2,099(2.2)$ & $195(2.1)$ & \\
\hline \multicolumn{7}{|l|}{ Control/ownership of hospital } & \multirow{4}{*}{$<0.001$} \\
\hline Government, nonfederal & $92,418(10.9)$ & $2,237(14.4)$ & $45,320(13.6)$ & $37,570(9.5)$ & $6,538(6.8)$ & $753(8.0)$ & \\
\hline Private, nonprofit & $645,943(76.1)$ & $10,437(67.2)$ & $235,140(70.7)$ & $312,587(79.2)$ & $80,227(83.6)$ & $7,552(80.0)$ & \\
\hline Private, investor-owned & $110,195(13.0)$ & $2,859(18.4)$ & $52,321(15.7)$ & $44,638(11.3)$ & $9,247(9.6)$ & $1,130(12.0)$ & \\
\hline \multicolumn{7}{|c|}{ Teaching status of urban hospitals } & \multirow{4}{*}{$<0.001$} \\
\hline Metropolitan nonteaching & $186,918(22.0)$ & $4,040(26.0)$ & $85,984(25.8)$ & $77,385(19.6)$ & $17,814(18.6)$ & $1,694(18.0)$ & \\
\hline Metropolitan teaching & $578,867(68.2)$ & $7,994(51.5)$ & $197,715(59.4)$ & $290,987(73.7)$ & $74,847(78.0)$ & 7,324 (77.6) & \\
\hline Nonmetropolitan hospital & $82,771(9.8)$ & $3,499(22.5)$ & $49,081(14.7)$ & $26,423(6.7)$ & $3,351(3.5)$ & $417(4.4)$ & \\
\hline \multicolumn{7}{|l|}{ Bed size of hospital } & \multirow{4}{*}{0.02} \\
\hline Small & $137,589(16.2)$ & $2,976(19.2)$ & $57,953(17.4)$ & $62,125(15.7)$ & $13,335(13.9)$ & $1,200(12.7)$ & \\
\hline Medium & $251,640(29.7)$ & $4,653(30.0)$ & $100,057(30.1)$ & $119,851(30.4)$ & $24,687(25.7)$ & $2,392(25.4)$ & \\
\hline Large & $459,327(54.1)$ & $7,904(50.9)$ & $174,772(52.5)$ & $212,819(53.9)$ & $57,990(60.4)$ & $5,843(61.9)$ & \\
\hline \multicolumn{7}{|c|}{ Zip code median household income $(\$ 1,000$ s) } & \multirow{5}{*}{$<0.001$} \\
\hline $1-43.9$ & $235,253(27.7)$ & $5,191(33.4)$ & $108,155(32.5)$ & $101,094(25.6)$ & $18,413(19.2)$ & $2,400(25.4)$ & \\
\hline $44.0-55.9$ & $232,559(27.4)$ & $4,979(32.1)$ & $100,272(30.1)$ & $103,549(26.2)$ & $21,291(22.2)$ & $2,467(26.1)$ & \\
\hline $56.0-73.9$ & $205,889(24.3)$ & $3,420(22.0)$ & $75,369(22.6)$ & $100,366(25.4)$ & $24,556(25.6)$ & $2,178(23.1)$ & \\
\hline $74.0+$ & $174,855(20.6)$ & $1,943(12.5)$ & $48,984(14.7)$ & $89,786(22.7)$ & $31,751(33.1)$ & $2,390(25.3)$ & \\
\hline $\begin{array}{l}\text { Number of patients } \\
\text { at facility in cohort }\end{array}$ & $717.1(775.3)$ & $472.6(513.8)$ & $587.6(578.9)$ & $780.8(819.1)$ & $898.2(1,011.7)$ & $1,178.9(1,438.0)$ & $<0.001$ \\
\hline \multicolumn{8}{|l|}{ Comorbid conditions } \\
\hline Placenta previa & $9,038(1.1)$ & $140(0.9)$ & $2,509(0.8)$ & $4,468(1.1)$ & $1,768(1.8)$ & $152(1.6)$ & $<0.001$ \\
\hline Gestational hypertension & $58,809(6.9)$ & $852(5.5)$ & $19,094(5.7)$ & $28,399(7.2)$ & $8,688(9.0)$ & $1,777(18.8)$ & $<0.001$ \\
\hline Preexisting hypertension & $28,547(3.4)$ & 454 (2.9) & $10,157(3.1)$ & $13,216(3.3)$ & $3,919(4.1)$ & $803(8.5)$ & $<0.001$ \\
\hline $\begin{array}{l}\text { Systemic lupus } \\
\text { erythematosus }\end{array}$ & $1,226(0.1)$ & $18(0.1)$ & $384(0.1)$ & $608(0.2)$ & $191(0.2)$ & $25(0.3)$ & $<0.001$ \\
\hline $\begin{array}{l}\text { Human immunodeficiency } \\
\text { virus }\end{array}$ & $1,300(0.2)$ & $15(0.1)$ & $355(0.1)$ & $754(0.2)$ & $161(0.2)$ & $16(0.2)$ & $<0.001$ \\
\hline Drug abuse & $21,348(2.5)$ & $563(3.6)$ & $7,417(2.2)$ & $9,280(2.4)$ & $3,554(3.7)$ & $535(5.7)$ & $<0.001$ \\
\hline Alcohol abuse & $1,038(0.1)$ & $30(0.2)$ & $349(0.1)$ & $477(0.1)$ & $146(0.2)$ & $35(0.4)$ & $<0.001$ \\
\hline Tobacco use & $50,069(5.9)$ & $1,491(9.6)$ & $23,642(7.1)$ & $19,461(4.9)$ & $4,817(5.0)$ & $658(7.0)$ & $<0.001$ \\
\hline Asthma & $45,630(5.4)$ & $658(4.2)$ & $15,339(4.6)$ & $22,335(5.7)$ & $6,543(6.8)$ & $754(8.0)$ & $<0.001$ \\
\hline $\begin{array}{l}\text { Preexisting diabetes } \\
\text { mellitus }\end{array}$ & $15,169(1.8)$ & $279(1.8)$ & $4,521(1.4)$ & $7,244(1.8)$ & $2,763(2.9)$ & $362(3.8)$ & $<0.001$ \\
\hline $\begin{array}{l}\text { Gestational diabetes } \\
\text { mellitus }\end{array}$ & $81,156(9.6)$ & $1,209(7.8)$ & $29,279(8.8)$ & $38,938(9.9)$ & $10,649(11.1)$ & $1,081(11.5)$ & $<0.001$ \\
\hline Obesity & $129,690(15.3)$ & $2,024(13.0)$ & $50,493(15.2)$ & $60,967(15.4)$ & $14,463(15.1)$ & $1,744(18.5)$ & 0.05 \\
\hline \multicolumn{8}{|c|}{ Indications for cesarean delivery } \\
\hline Previous cesarean delivery & $441,358(52.0)$ & $9,107(58.6)$ & $199,872(60.1)$ & $190,793(48.3)$ & $38,840(40.5)$ & $2,745(29.1)$ & $<0.001$ \\
\hline Fetal malpresentation & $124,550(14.7)$ & 1,964 (12.6) & $43,348(13.0)$ & $61,104(15.5)$ & $16,725(17.4)$ & $1,410(14.9)$ & $<0.001$ \\
\hline
\end{tabular}


e192 Hospitalization Following Cesarean Delivery Federspiel et al.

Table 1 (Continued)

\begin{tabular}{|c|c|c|c|c|c|c|c|}
\hline \multirow[b]{3}{*}{$\begin{array}{l}\text { Fetal hydrocephalus } \\
\text { or CNS malformation }\end{array}$} & \multicolumn{7}{|c|}{ Length of stay following cesarean delivery (d) } \\
\hline & \multirow{2}{*}{$\begin{array}{l}\text { Overall } \\
(N=456,312) \\
\text { (weighted } \\
N=848,556)\end{array}$} & \multirow{2}{*}{$\begin{array}{l}<2 \mathrm{~d} \\
(N=8,276) \\
\text { (weighted } \\
N=15,533)\end{array}$} & \multirow{2}{*}{$\begin{array}{l}2 \mathrm{~d} \\
(N=178,474) \\
\text { (weighted } \\
N=332,781)\end{array}$} & \multirow{2}{*}{$\begin{array}{l}\mathrm{d} \\
(N=211,605) \\
\text { (weighted } \\
N=394,795)\end{array}$} & \multirow{2}{*}{$\begin{array}{l}\mathrm{d} \\
(N=52,693) \\
\text { (weighted } \\
N=96,012)\end{array}$} & \multirow{2}{*}{$\begin{array}{l}>4 \mathrm{~d} \\
(N=5,264) \\
\text { (weighted } \\
N=9,435)\end{array}$} & \multirow{2}{*}{$\begin{array}{l}p \text {-Value } \\
1,111 \\
(0.1)\end{array}$} \\
\hline & & & & & & & \\
\hline $40(0.3)$ & $293(0.1)$ & $526(0.1)$ & $235(0.2)$ & $17(0.2)$ & $<0.001$ & & \\
\hline Fetal distress & $174,619(20.6)$ & $2,946(19.0)$ & $53,565(16.1)$ & $88,441(22.4)$ & $26,365(27.5)$ & $3,301(35.0)$ & $<0.001$ \\
\hline Failed operative delivery & $4,952(0.6)$ & $64(0.4)$ & $1,350(0.4)$ & $2,522(0.6)$ & $922(1.0)$ & $93(1.0)$ & $<0.001$ \\
\hline Cord prolapse & $3,981(0.5)$ & $83(0.5)$ & $1,266(0.4)$ & $1,991(0.5)$ & $555(0.6)$ & $86(0.9)$ & $<0.001$ \\
\hline Vasa previa & $921(0.1)$ & $16(0.1)$ & $156(0.0)$ & $432(0.1)$ & $288(0.3)$ & $30(0.3)$ & $<0.001$ \\
\hline $\begin{array}{l}\text { Fetal-maternal } \\
\text { disproportion }\end{array}$ & $23,692(2.8)$ & $355(2.3)$ & $8,810(2.6)$ & $11,362(2.9)$ & $2,839(3.0)$ & $325(3.4)$ & 0.02 \\
\hline Obstructed labor & $23,641(2.8)$ & $435(2.8)$ & $8,228(2.5)$ & $11,431(2.9)$ & $3,228(3.4)$ & $319(3.4)$ & $<0.001$ \\
\hline Abnormal forces of labor & $120,032(14.1)$ & $1,696(10.9)$ & $36,636(11.0)$ & $62,569(15.8)$ & $16,699(17.4)$ & $2,432(25.8)$ & $<0.001$ \\
\hline Long labor & $9,404(1.1)$ & $126(0.8)$ & $2,858(0.9)$ & $4,633(1.2)$ & $1,579(1.6)$ & $208(2.2)$ & $<0.001$ \\
\hline Failed induction & $31,444(3.7)$ & $387(2.5)$ & $9,883(3.0)$ & $16,096(4.1)$ & $4,151(4.3)$ & $927(9.8)$ & $<0.001$ \\
\hline $\begin{array}{l}\text { Intrauterine } \\
\text { growth restriction }\end{array}$ & $27,809(3.3)$ & $393(2.5)$ & $8,258(2.5)$ & $13,383(3.4)$ & $5,097(5.3)$ & $678(7.2)$ & $<0.001$ \\
\hline Macrosomia & $46,608(5.5)$ & 609 (3.9) & $15,831(4.8)$ & $23,659(6.0)$ & $5,994(6.2)$ & $514(5.4)$ & $<0.001$ \\
\hline HSV infection & $14,117(1.7)$ & $176(1.1)$ & $4,682(1.4)$ & $7,107(1.8)$ & $1,925(2.0)$ & $228(2.4)$ & $<0.001$ \\
\hline Oligohydramnios & $28,061(3.3)$ & $372(2.4)$ & $8,883(2.7)$ & $14,102(3.6)$ & $4,140(4.3)$ & $565(6.0)$ & $<0.001$ \\
\hline Other fetal anomalies & $2,387(0.3)$ & $27(0.2)$ & $807(0.2)$ & $1,143(0.3)$ & $371(0.4)$ & $39(0.4)$ & $<0.001$ \\
\hline Vaginal anomalies & $383(0.0)$ & $(0.0)^{\mathrm{a}}$ & $134(0.0)$ & $194(0.0)$ & $45(0.0)$ & $(0.1)^{\mathrm{a}}$ & 0.58 \\
\hline \multicolumn{8}{|l|}{ Outcomes } \\
\hline $\begin{array}{l}\text { Any readmission } \\
\text { within } 30 \mathrm{~d}\end{array}$ & $14,059(1.7)$ & $223(1.4)$ & $4,680(1.4)$ & $6,805(1.7)$ & $1,942(2.0)$ & 409 (4.3) & $<0.001$ \\
\hline $\begin{array}{l}\text { Total inpatient } \\
\text { charges }(\$ 1,000 \text { s) }\end{array}$ & $25.6(19.5)$ & $23.1(81.7)$ & $23.4(13.3)$ & $26.1(15.4)$ & $30.3(21.3)$ & $44.4(39.4)$ & $<0.001$ \\
\hline $\begin{array}{l}\text { Total inpatient } \\
\text { costs }(\$ 1,000 \mathrm{~s})\end{array}$ & $6.8(6.2)$ & $6.2(34.5)$ & $6.1(3.5)$ & $6.9(3.9)$ & $8.4(5.2)$ & $11.7(9.2)$ & $<0.001$ \\
\hline
\end{tabular}

Abbreviations: CNS, central nervous system; HSV, herpes simplex virus.

Note: $p$-Values by weighted linear regression for continuous variables and weighted chi-square test for binary/categorical variables. Missing values in charges and costs (27 observations each).

${ }^{\mathrm{a}} \leq=10$; exact value suppressed due to privacy protections from data supplier.

rates of postoperative day 2 or earlier discharge were not associated with rates of all-cause 30-day readmission but were associated with lower costs.

This study evaluated risk factors to further explore differences in length of stays less than 4 days after cesarean delivery in the United States, whereas previous studies focused primarily on length of discharge more than 4 days ${ }^{11}$ or did not focus specifically on cesarean deliveries. ${ }^{12}$ We found within our low-risk cohort that in general, younger patients with fewer comorbidities and a history of prior cesarean delivery were more likely to be discharged in 2 days or less, which was an expected finding. History of cesarean delivery was a positive predictor of 2-day discharge, whereas most of the cesarean indications suggestive of labored cesarean section (such as failed induction or abnormal forces of labor) were negative predictors of 2-day discharge. This provides indirect evidence that patients presenting for scheduled cesarean delivery appear more likely to have 2-day discharge when compared with those having unlabored cesarean deliveries. There were interesting intersections between insurance status and ZIP code-level income in that after adjustment, Medicaid recipients were less likely to be discharged home on the second hospital day, while patients from lower income ZIP codes were more likely to be discharged home on the second hospital day. This discrepancy may reflect differences in the unit of measurement (individual patients vs. ZIP code), or more interactions between income and insurance carrier (e.g., that patients who are covered by Medicaid in high-income ZIP codes differ from those residing in low-income ZIP codes). Further research with individual-level income and insurance data would be needed to clarify this dynamic.

Prior studies on length of stay have focused on effects of early discharge and risk factors for early discharge. A 2002 Cochrane review indicated no difference in infant readmission for an early postnatal discharge and no pooled difference in maternal readmissions, although definitions of early discharge varied widely and most included patients with vaginal rather than cesarean deliveries. ${ }^{13}$ An observational study in Egypt also noted no difference in maternal hospital readmissions if patients were discharged at 24 versus 72 hours following cesarean section. ${ }^{14}$ These studies are in 
Table 2 Adjusted predictors of a 2-day or shorter hospital stay following cesarean delivery

\begin{tabular}{|c|c|}
\hline & $\begin{array}{l}\text { Relative risk ( } 95 \% \\
\text { confidence interval) }\end{array}$ \\
\hline Age in y at admission & $0.99^{\mathrm{a}}(0.98,0.99)$ \\
\hline \multicolumn{2}{|l|}{ Primary payer (vs. private) } \\
\hline Medicare & $0.75^{\mathrm{a}}(0.68,0.82)$ \\
\hline Medicaid & $0.88^{\mathrm{a}}(0.85,0.91)$ \\
\hline Self-pay & $1.07(0.98,1.17)$ \\
\hline No charge & $0.77(0.52,1.14)$ \\
\hline Other & $1.08^{\mathrm{b}}(1.02,1.15)$ \\
\hline \multicolumn{2}{|c|}{ Control/ownership of hospital (vs. government, nonfederal) } \\
\hline Private, nonprofit & $0.81^{\mathrm{a}}(0.72,0.90)$ \\
\hline Private, investor-owned & $0.95(0.83,1.09)$ \\
\hline \multicolumn{2}{|l|}{$\begin{array}{l}\text { Teaching status of urban hospitals } \\
\text { (vs. metropolitan, nonteaching) }\end{array}$} \\
\hline Metropolitan teaching & $0.80^{\mathrm{a}}(0.74,0.87)$ \\
\hline Nonmetropolitan hospital & $1.21^{\mathrm{a}}(1.12,1.32)$ \\
\hline \multicolumn{2}{|l|}{ Bed size of hospital (vs. small) } \\
\hline Medium & $0.95(0.86,1.04)$ \\
\hline Large & $0.93(0.84,1.02)$ \\
\hline \multicolumn{2}{|c|}{ Zip code median household income $(\$ 1,000$ s) (vs. \$1-43.9) } \\
\hline$\$ 44.0-55.9$ & $0.95^{\mathrm{c}}(0.91,0.98)$ \\
\hline $56.0-73.9$ & $0.85^{\mathrm{a}}(0.81,0.89)$ \\
\hline $74.0+$ & $0.69^{\mathrm{a}}(0.63,0.76)$ \\
\hline $\begin{array}{l}\text { Number of patients } \\
\text { at facility in cohort }\end{array}$ & $1.00^{\mathrm{b}}(1.00,1.00)$ \\
\hline \multicolumn{2}{|l|}{ Comorbid conditions } \\
\hline Placenta previa & $0.81^{\mathrm{a}}(0.77,0.86)$ \\
\hline Gestational hypertension & $0.85^{\mathrm{a}}(0.83,0.87)$ \\
\hline Preexisting hypertension & $0.92^{\mathrm{a}}(0.89,0.95)$ \\
\hline Systemic lupus erythematosus & $0.88^{\mathrm{b}}(0.79,0.98)$ \\
\hline Human immunodeficiency virus & $0.70^{\mathrm{a}}(0.59,0.83)$ \\
\hline Drug Abuse & $0.79^{\mathrm{a}}(0.75,0.83)$ \\
\hline Alcohol abuse & $0.97(0.83,1.14)$ \\
\hline Tobacco use & $1.18^{\mathrm{a}}(1.14,1.21)$ \\
\hline Asthma & $0.89^{a}(0.86,0.92)$ \\
\hline Preexisting diabetes mellitus & $0.78^{\mathrm{a}}(0.75,0.82)$ \\
\hline Gestational diabetes mellitus & $0.94^{\mathrm{a}}(0.92,0.96)$ \\
\hline Obesity & $1.01(0.98,1.05)$ \\
\hline \multicolumn{2}{|l|}{ Indications for cesarean delivery } \\
\hline Previous cesarean delivery & $1.29^{\mathrm{a}}(1.26,1.32)$ \\
\hline Fetal malpresentation & $0.95^{\mathrm{a}}(0.93,0.97)$ \\
\hline $\begin{array}{l}\text { Fetal hydrocephalus } \\
\text { or CNS malformation }\end{array}$ & $0.86(0.73,1.01)$ \\
\hline Fetal distress & $0.88^{\mathrm{a}}(0.85,0.90)$ \\
\hline Failed operative delivery & $0.84^{\mathrm{a}}(0.78,0.91)$ \\
\hline
\end{tabular}

(Continued)
Table 2 (Continued)

\begin{tabular}{|l|l|}
\hline & $\begin{array}{l}\text { Relative risk }(95 \% \\
\text { confidence interval) }\end{array}$ \\
\hline Cord Prolapse & $0.93^{\mathrm{b}}(0.87,0.99)$ \\
\hline Vasa previa & $0.57^{\mathrm{a}}(0.47,0.69)$ \\
\hline Fetal-maternal disproportion & $0.98(0.94,1.03)$ \\
\hline Obstructed labor & $0.95^{\mathrm{b}}(0.91,1.00)$ \\
\hline Abnormal forces of labor & $0.87^{\mathrm{a}}(0.85,0.89)$ \\
\hline Long labor & $0.95^{(0.90,1.01)}$ \\
\hline Failed induction & $0.94^{\mathrm{a}}(0.91,0.97)$ \\
\hline Intrauterine growth restriction & $0.80^{\mathrm{a}}(0.77,0.83)$ \\
\hline Macrosomia & $0.92^{\mathrm{a}}(0.90,0.95)$ \\
\hline HSV infection & $0.89^{\mathrm{a}}(0.84,0.93)$ \\
\hline Oligohydramnios & $0.91^{\mathrm{a}}(0.88,0.94)$ \\
\hline Other fetal anomalies & $1.01^{(0.92,1.11)}$ \\
\hline Vaginal anomalies & $0.97^{(0.80,1.19)}$ \\
\hline
\end{tabular}

Abbreviation: CNS, central nervous system; HSV, herpes simplex virus. ${ }^{\mathrm{a}} p<0.001$.

$\mathrm{b}_{p}<0.05$.

${ }^{c} p<0.01$.

accord with our findings of no increased rate of readmission within 30 days based on length of stay. In contrast, a Canadian study in 2002 reported increased maternal readmissions with length of stays less than 4 days, when compared with 5 days. ${ }^{15}$ We note the difference in using 4 versus 2 days as the breakpoint in their analysis when compared with our own. Given that postpartum readmissions in general are rare in the United States, occurring in only $1.01 \%$ of all patients, and only $1.7 \%$ in our low risk, cesarean delivery only sample, it may be more difficult to find significant differences in readmission rates. ${ }^{16}$ From a global perspective, a review of length of stay after cesarean delivery in 92 low- to middle-income countries described large variability with mean lengths of stay of 2.5 to 9.3 days. ${ }^{17}$

The significant variation in postcesarean length of stay at the facility level in this study is consistent with an extensive body of literature in the United States documenting geographic and provider-level variations in care. ${ }^{6,18}$ In the case of length of stay after an uncomplicated cesarean delivery, based on our anecdotal experiences in different facilities, we suspect that a component of the facility-level variation may reflect an ingrained institutional habit (i.e., "how we have always done this") in terms of when discharge is offered to patients. Our results should provide some reassurance that in a subset of low-risk patients, high facility-level rates of discharge within 2 days of cesarean delivery was not associated with readmissions. These results suggest that offering discharge on postoperative day 2 , or discharging patients on postoperative day 2 on their request, may be reasonable in appropriately selected patients.

This study's results were enhanced by several strengths. The use of the 2017 NRD provides a large, contemporary sample of more than $50 \%$ of all U.S. hospitalizations and more 

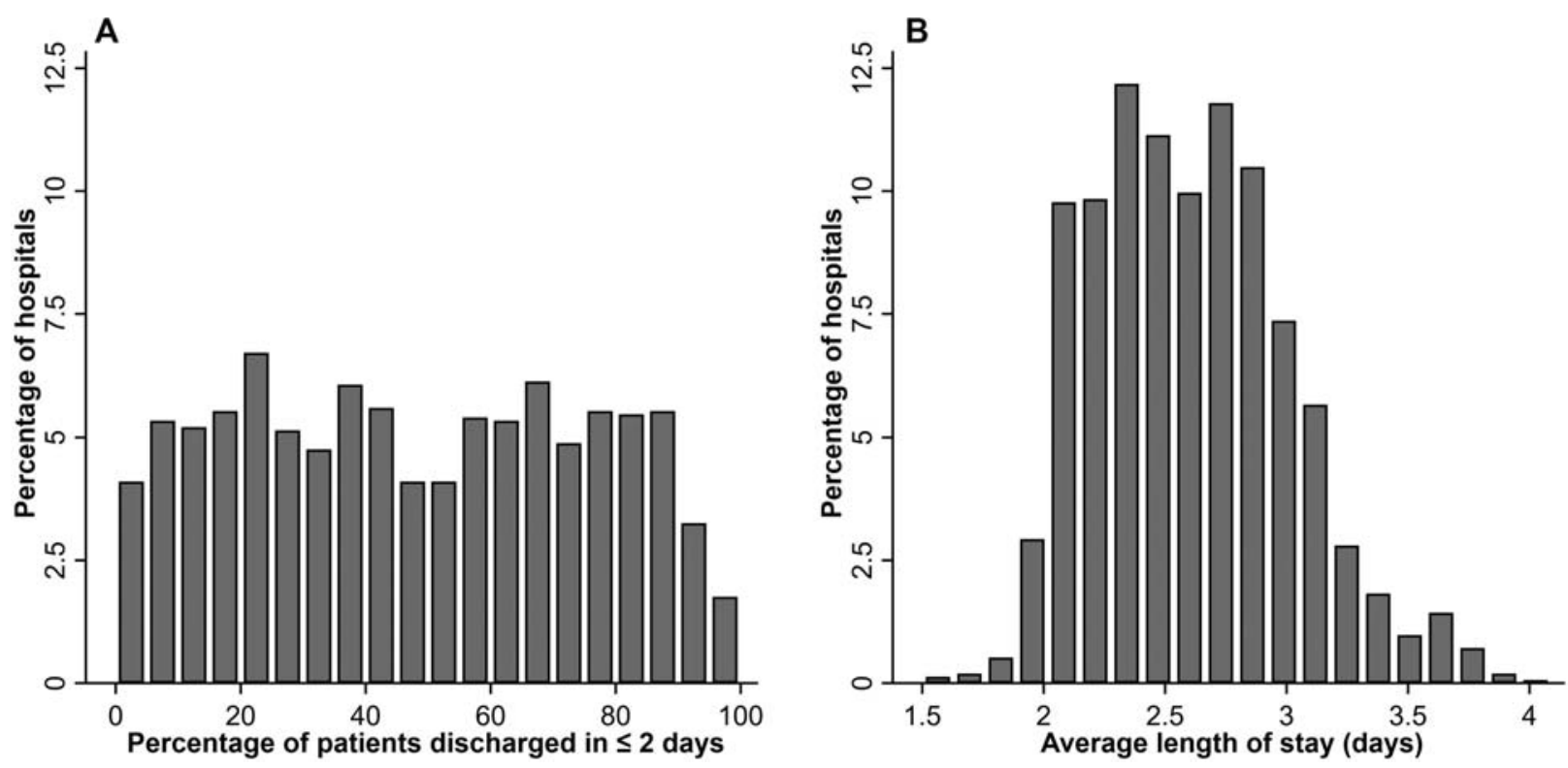

Fig. 2 (A) Facility variation in the percentage of patients discharged at or before postoperative day 2. Graph depicts the number of hospitals (as a percentage) that discharge patients on postoperative day 2 or sooner, when expressed as a percentage of their total postcesarean discharges. (B) Facility variation in average length of stay in days.

Table 3 Baseline patient and facility characteristics, stratified by facility percentage of patients discharged within 2 days of cesarean delivery

\begin{tabular}{|c|c|c|c|c|c|c|}
\hline & \multicolumn{6}{|c|}{ Percentage of patients discharged in $2 \mathrm{~d}$ or less (quartiles) } \\
\hline & $\begin{array}{l}\text { Overall } \\
(N=456,312) \\
\text { (weighted } \\
N=848,556)\end{array}$ & $\begin{array}{l}0.0 \%-17.8 \% \\
(N=114,377) \\
\text { (weighted } \\
N=200,456)\end{array}$ & $\begin{array}{l}17.8-38.1 \% \\
(N=114,344) \\
\text { (weighted } \\
N=225,166)\end{array}$ & $\begin{array}{l}38.2-63.3 \% \\
(N=113,865) \\
\text { (weighted } \\
N=217,095)\end{array}$ & $\begin{array}{l}63.3-100.0 \% \\
(N=113,726) \\
\text { (weighted } \\
N=205,839)\end{array}$ & $p$-Value \\
\hline \multicolumn{7}{|l|}{ Mean (standard deviation) or $\%$} \\
\hline Age in $y$ at admission & $29.9(5.7)$ & $31.3(5.8)$ & $30.1(5.4)$ & $29.5(5.6)$ & $28.6(5.8)$ & $<0.001$ \\
\hline \multicolumn{7}{|l|}{ Primary payer } \\
\hline Medicare & $7,953(0.9)$ & $2,382(1.2)$ & $1,598(0.7)$ & $2,332(1.1)$ & $1,641(0.8)$ & \multirow[t]{6}{*}{$<0.001$} \\
\hline Medicaid & $349,491(41.2)$ & $74,611(37.2)$ & $86,208(38.3)$ & $89,829(41.4)$ & $98,843(48.0)$ & \\
\hline Private & $457,887(54.0)$ & $118,128(58.9)$ & $128,041(56.9)$ & $115,713(53.3)$ & $96,004(46.6)$ & \\
\hline Self-pay & $10,281(1.2)$ & $2,469(1.2)$ & $2,544(1.1)$ & $2,653(1.2)$ & $2,615(1.3)$ & \\
\hline No charge & $416(0.0)$ & $105(0.1)$ & $138(0.1)$ & $99(0.0)$ & $74(0.0)$ & \\
\hline Other & $22,529(2.7)$ & $2,761(1.4)$ & $6,637(2.9)$ & $6,470(3.0)$ & $6,662(3.2)$ & \\
\hline \multicolumn{7}{|l|}{ Control/ownership of hospital } \\
\hline Government, nonfederal & $92,418(10.9)$ & $16,347(8.2)$ & $16,698(7.4)$ & $21,333(9.8)$ & $38,040(18.5)$ & \multirow[t]{3}{*}{$<0.001$} \\
\hline Private, nonprofit & $645,943(76.1)$ & $171,214(85.4)$ & $179,074(79.5)$ & $170,145(78.4)$ & $125,511(61.0)$ & \\
\hline Private, investor-owned & $110,195(13.0)$ & $12,894(6.4)$ & 29,395 (13.1) & $25,617(11.8)$ & $42,289(20.5)$ & \\
\hline \multicolumn{7}{|c|}{ Teaching status of urban hospitals } \\
\hline Metropolitan nonteaching & $186,918(22.0)$ & $27,030(13.5)$ & $46,844(20.8)$ & $53,150(24.5)$ & $59,894(29.1)$ & \multirow[t]{3}{*}{$<0.001$} \\
\hline Metropolitan teaching & $578,867(68.2)$ & $170,728(85.2)$ & $165,877(73.7)$ & $141,216(65.0)$ & $101,047(49.1)$ & \\
\hline Nonmetropolitan hospital & $82,771(9.8)$ & $2,698(1.3)$ & $12,445(5.5)$ & $22,730(10.5)$ & $44,898(21.8)$ & \\
\hline \multicolumn{7}{|l|}{ Bed size of hospital } \\
\hline Small & $137,589(16.2)$ & $25,635(12.8)$ & $41,247(18.3)$ & $31,005(14.3)$ & $39,702(19.3)$ & \multirow[t]{3}{*}{0.01} \\
\hline Medium & $251,640(29.7)$ & $50,317(25.1)$ & $81,885(36.4)$ & $52,564(24.2)$ & $66,874(32.5)$ & \\
\hline Large & $459,327(54.1)$ & $124,504(62.1)$ & $102,035(45.3)$ & $133,526(61.5)$ & $99,263(48.2)$ & \\
\hline \multicolumn{7}{|c|}{ Zip code median household income $(\$ 1,000$ s) } \\
\hline $1-43.9$ & $235,253(27.7)$ & $38,894(19.4)$ & $49,728(22.1)$ & $64,500(29.7)$ & $82,131(39.9)$ & \multirow[t]{3}{*}{$<0.001$} \\
\hline $44.0-55.9$ & $232,559(27.4)$ & $39,659(19.8)$ & $58,486(26.0)$ & $69,084(31.8)$ & $65,330(31.7)$ & \\
\hline $56.0-73.9$ & 205,889 (24.3) & $49,081(24.5)$ & $65,953(29.3)$ & $52,706(24.3)$ & $38,150(18.5)$ & \\
\hline
\end{tabular}


Table 3 (Continued)

\begin{tabular}{|c|c|c|c|c|c|c|}
\hline & \multicolumn{6}{|c|}{ Percentage of patients discharged in $2 \mathrm{~d}$ or less (quartiles) } \\
\hline & $\begin{array}{l}\text { Overall } \\
(N=456,312) \\
\text { (weighted } \\
N=848,556)\end{array}$ & $\begin{array}{l}0.0 \%-17.8 \% \\
(N=114,377) \\
\text { (weighted } \\
N=200,456)\end{array}$ & $\begin{array}{l}17.8-38.1 \% \\
(N=114,344) \\
\text { (weighted } \\
N=225,166)\end{array}$ & $\begin{array}{l}38.2-63.3 \% \\
(N=113,865) \\
\text { (weighted } \\
N=217,095)\end{array}$ & $\begin{array}{l}63.3-100.0 \% \\
(N=113,726) \\
\text { (weighted } \\
N=205,839)\end{array}$ & $p$-Value \\
\hline $74.0+$ & $174,855(20.6)$ & $72,822(36.3)$ & $50,999(22.6)$ & $30,805(14.2)$ & $20,228(9.8)$ & \\
\hline $\begin{array}{l}\text { Number of patients at } \\
\text { facility in cohort }\end{array}$ & $717.1(775.3)$ & $1,042.3(1,201.8)$ & $652.0(478.0)$ & $759.4(730.3)$ & $427.0(347.3)$ & $<0.001$ \\
\hline \multicolumn{7}{|l|}{ Comorbid conditions } \\
\hline Placenta previa & $9,038(1.1)$ & $2,625(1.3)$ & $2,404(1.1)$ & $2,200(1.0)$ & $1,809(0.9)$ & $<0.001$ \\
\hline Gestational hypertension & $58,809(6.9)$ & $13,156(6.6)$ & $16,027(7.1)$ & $15,674(7.2)$ & $13,952(6.8)$ & 0.13 \\
\hline Preexisting hypertension & $28,547(3.4)$ & $6,043(3.0)$ & $7,427(3.3)$ & $7,891(3.6)$ & $7,186(3.5)$ & 0.01 \\
\hline Systemic lupus erythematosus & $1,226(0.1)$ & $358(0.2)$ & $330(0.1)$ & $314(0.1)$ & $223(0.1)$ & 0.003 \\
\hline Human immunodeficiency virus & $1,300(0.2)$ & $286(0.1)$ & $370(0.2)$ & $370(0.2)$ & $274(0.1)$ & 0.76 \\
\hline Drug abuse & $21,348(2.5)$ & $3,695(1.8)$ & $5,427(2.4)$ & $5,691(2.6)$ & $6,536(3.2)$ & $<0.001$ \\
\hline Alcohol abuse & $1,038(0.1)$ & $214(0.1)$ & $293(0.1)$ & $251(0.1)$ & $280(0.1)$ & 0.48 \\
\hline Tobacco use & $50,069(5.9)$ & $6,603(3.3)$ & $13,033(5.8)$ & $14,491(6.7)$ & $15,942(7.7)$ & $<0.001$ \\
\hline Asthma & $45,630(5.4)$ & $12,275(6.1)$ & $12,758(5.7)$ & $11,482(5.3)$ & $9,114(4.4)$ & $<0.001$ \\
\hline Preexisting diabetes mellitus & $15,169(1.8)$ & $3,476(1.7)$ & $4,128(1.8)$ & $4,101(1.9)$ & $3,464(1.7)$ & 0.32 \\
\hline Gestational diabetes mellitus & $81,156(9.6)$ & $19,999(10.0)$ & $22,077(9.8)$ & $20,221(9.3)$ & $18,860(9.2)$ & 0.02 \\
\hline Obesity & $129,690(15.3)$ & $26,900(13.4)$ & $34,609(15.4)$ & $35,823(16.5)$ & $32,359(15.7)$ & 0.03 \\
\hline \multicolumn{7}{|l|}{ Indications for cesarean delivery } \\
\hline Previous cesarean delivery & $441,358(52.0)$ & $100,948(50.4)$ & $118,018(52.4)$ & $113,746(52.4)$ & $108,646(52.8)$ & $<0.001$ \\
\hline Fetal malpresentation & $124,550(14.7)$ & $30,743(15.3)$ & $34,697(15.4)$ & $30,939(14.3)$ & $28,171(13.7)$ & $<0.001$ \\
\hline $\begin{array}{l}\text { Fetal hydrocephalus } \\
\text { or CNS malformation }\end{array}$ & $1,111(0.1)$ & $287(0.1)$ & $290(0.1)$ & $375(0.2)$ & $159(0.1)$ & 0.03 \\
\hline Fetal distress & $174,619(20.6)$ & $46,727(23.3)$ & $45,055(20.0)$ & $43,250(19.9)$ & $39,588(19.2)$ & $<0.001$ \\
\hline Failed operative delivery & $4,952(0.6)$ & $1,209(0.6)$ & $1,438(0.6)$ & $1,144(0.5)$ & $1,161(0.6)$ & 0.16 \\
\hline Cord prolapse & $3,981(0.5)$ & $901(0.4)$ & $1,059(0.5)$ & $1,036(0.5)$ & $985(0.5)$ & 0.88 \\
\hline Vasa previa & $921(0.1)$ & $299(0.1)$ & $271(0.1)$ & $230(0.1)$ & $121(0.1)$ & $<0.001$ \\
\hline Fetal-maternal disproportion & $23,692(2.8)$ & $3,972(2.0)$ & $6,642(2.9)$ & $5,939(2.7)$ & $7,139(3.5)$ & $<0.001$ \\
\hline Obstructed labor & $23,641(2.8)$ & $5,332(2.7)$ & $6,559(2.9)$ & $5,495(2.5)$ & $6,255(3.0)$ & 0.06 \\
\hline Abnormal forces of labor & $120,032(14.1)$ & $30,622(15.3)$ & $32,059(14.2)$ & $29,624(13.6)$ & $27,727(13.5)$ & $<0.001$ \\
\hline Long labor & $9,404(1.1)$ & $2,402(1.2)$ & $2,448(1.1)$ & $2,470(1.1)$ & $2,084(1.0)$ & 0.45 \\
\hline Failed induction & $31,444(3.7)$ & $8,125(4.1)$ & $8,136(3.6)$ & $7,998(3.7)$ & $7,186(3.5)$ & 0.08 \\
\hline Intrauterine growth restriction & $27,809(3.3)$ & $6,755(3.4)$ & $7,537(3.3)$ & $7,133(3.3)$ & $6,384(3.1)$ & 0.44 \\
\hline Macrosomia & $46,608(5.5)$ & $11,144(5.6)$ & $13,090(5.8)$ & $11,800(5.4)$ & $10,574(5.1)$ & 0.05 \\
\hline HSV infection & $14,117(1.7)$ & $3,646(1.8)$ & $3,951(1.8)$ & $3,501(1.6)$ & $3,018(1.5)$ & 0.13 \\
\hline Oligohydramnios & $28,061(3.3)$ & $7,538(3.8)$ & $7,063(3.1)$ & $6,886(3.2)$ & $6,575(3.2)$ & 0.001 \\
\hline Other fetal anomalies & $2,387(0.3)$ & $690(0.3)$ & $696(0.3)$ & $561(0.3)$ & $439(0.2)$ & 0.17 \\
\hline Vaginal anomalies & $383(0.0)$ & $97(0.0)$ & $122(0.1)$ & $86(0.0)$ & $79(0.0)$ & 0.43 \\
\hline \multicolumn{7}{|l|}{ Outcomes } \\
\hline Any readmission within $30 \mathrm{~d}$ & $14,059(1.7)$ & $3,333(1.7)$ & $3,753(1.7)$ & $3,476(1.6)$ & $3,496(1.7)$ & 0.67 \\
\hline Total inpatient charges $(\$ 1,000 \mathrm{~s})$ & $25.6(19.5)$ & $27.4(19.3)$ & $23.9(14.7)$ & $25.9(26.4)$ & $25.6(14.9)$ & 0.08 \\
\hline Total inpatient costs $(\$ 1,000$ s) & $6.8(6.2)$ & $7.5(4.5)$ & $6.5(3.5)$ & $6.8(10.1)$ & $6.5(4.0)$ & 0.001 \\
\hline
\end{tabular}

Abbreviation: CNS, central nervous system; HSV, herpes simplex virus.

Note: $p$-Values by weighted linear regression for continuous variables and weighted chi-square test for binary/categorical variables. Missing values in charges and costs (27 observations each). 


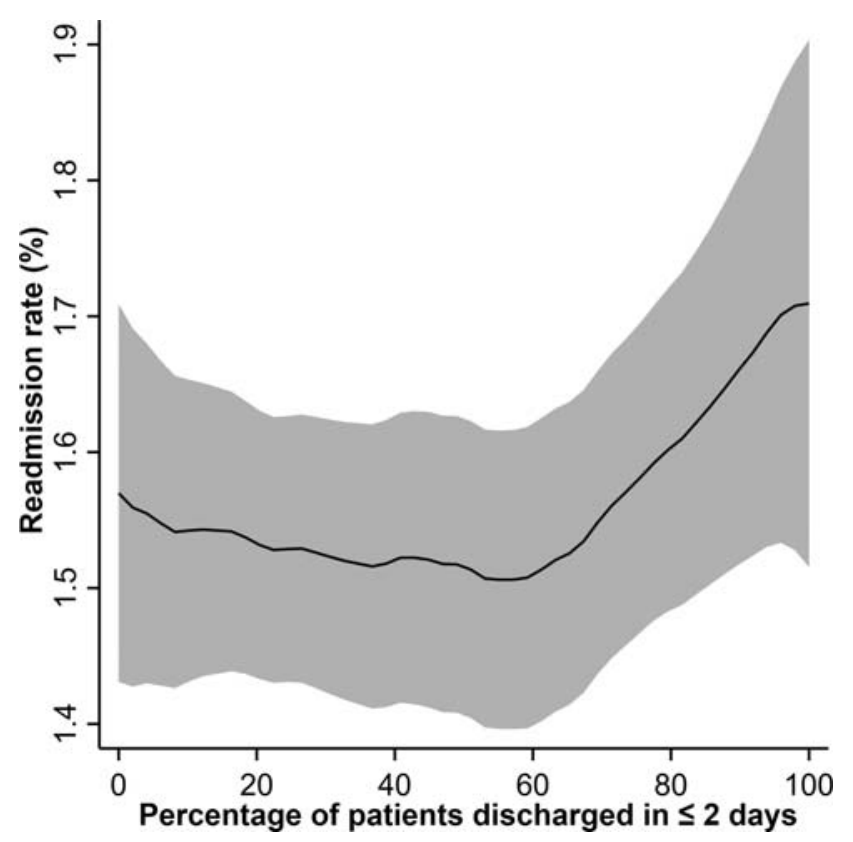

Fig. 3 Unadjusted association between facility-level rate of discharge within 2 days of cesarean delivery and 30-day, all-cause readmission rate. Facilities are classified along the $X$-axis according to the percentage of their patients that are discharged within 2 days of cesarean. The 30 -day readmission rates are expressed as percentage with $95 \%$ confidence intervals.

than 1,500 facilities. This is a large sample, designed for use in generating nationally representative estimates of readmissions. The NRD includes both patient and facility factors, providing a diverse set of variables for consideration in analysis. However, as with all analyses, this study included weaknesses as well. The use of administrative data significantly limits the quality of covariate adjustment available, and the potential for confounding cannot be excluded. This analysis was deliberately limited to a small low-risk subset of all cesarean deliveries and does not reflect patients with high-risk features such as pregnancy-related hypertensive disorders or diabetes. We could not assess neonatal outcomes, including neonatal readmission, which is an important consideration in early maternal discharge. Cost data were generated by facility-wide cost-to-charge ratios rather than department-specific ratios, and are thus potentially less accurate, and reflect the hospital fees alone (do not reflect professional fees such as obstetrics and anesthesiology services). The use of ZIP code-level income has been found to be suboptimal, given the significant variation in income across many ZIP codes. ${ }^{19}$ Furthermore, other important considerations play a role in discharge timing after a cesarean delivery, including effective pain control, need for social services, breastfeeding, and postpartum education, which were not addressed in this study.

In conclusion, in this analysis of low-risk cesarean deliveries in the United States, significant variation in practice patterns existed in postcesarean section length of stay even after statistical adjustment. This variation was not associated with significant differences in 30-day readmission rates but was associated with lower total inpatient costs. Further study is necessary to better characterize postoperative processes of care, identification of which patients may be candidates for earlier discharge, and the implications of earlier discharge on maternal and neonatal health, economics, and patient satisfaction.

Note

A preliminary version of this report was presented in poster form at the 38th Annual Society for Maternal-Fetal Medicine Annual Pregnancy Meeting (January 29February 3, 2018 in Dallas, TX).

This research was funded by a Kelly Resident Research Award from the Department of Gynecology and Obstetrics, Johns Hopkins School of Medicine. The funding source was not involved in the conduct of this research or preparation of this report.

\section{Conflict of Interest}

None declared.

\section{Acknowledgments}

This research was funded by a Howard A. Kelly Resident Research Grant from the Department of Gynecology and Obstetrics, Johns Hopkins University School of Medicine. The funding organization had no involvement in the design, conduct, or publication of this study.

The Nationwide Readmissions Database is a product of the Healthcare Cost and Utilization Project (HCUP) of the Agency for Healthcare Research and Quality. Partner organizations which contribute to HCUP datasets are listed at www.hcup-us.ahrq.gov/hcupdatapartners.jsp.

\section{References}

1 Cowper PA, DeLong ER, Hannan EL, et al. Trends in postoperative length of stay after bypass surgery. Am Heart J 2006;152(06): 1194-1200

2 Doll KM, Dusetzina SB, Robinson W. Trends in inpatient and outpatient hysterectomy and oophorectomy rates among commercially insured women in the United States, 2000-2014. JAMA Surg 2016;151(09):876-877

3 Mennuti MT. The shrinking postpartum hospital stay. Hosp Pract (1995) 1995;30(08):11-12

4 Lord M. Check in, deliver, go home. US News World Rep 1994;117 (22):98-100

5 Dahlke JD, Mendez-Figueroa H, Rouse DJ, Berghella V, Baxter JK, Chauhan SP. Evidence-based surgery for cesarean delivery: an updated systematic review. Am J Obstet Gynecol 2013;209(04): 294-306

6 Fisher ES, Wennberg DE, Stukel TA, Gottlieb DJ, Lucas FL, Pinder EL. The implications of regional variations in Medicare spending. Part 1: the content, quality, and accessibility of care. Ann Intern Med 2003;138(04):273-287

7 HCUP Nationwide Readmissions Database (NRS). Healthcare Cost and Utilization Project (HCUP). 2017. Agency for Healthcare Research and Quality, Rockville, MD. www.hcup-us.ahrq.gov/ nrdoverview.jsp

8 Bateman BT, Mhyre JM, Hernandez-Diaz S, et al. Development of a comorbidity index for use in obstetric patients. Obstet Gynecol 2013;122(05):957-965

9 Armstrong JC, Kozhimannil KB, McDermott P, Saade GR, Srinivas SK; Society for Maternal-Fetal Medicine Health Policy Committee. Comparing variation in hospital rates of cesarean delivery among 
low-risk women using 3 different measures. Am J Obstet Gynecol 2016;214(02):153-163

10 Henry OA, Gregory KD, Hobel CJ, Platt LD. Using ICD-9 codes to identify indications for primary and repeat cesarean sections: agreement with clinical records. Am J Public Health 1995;85(8 Pt 1):1143-1146

11 Blumenfeld YJ, El-Sayed YY, Lyell DJ, Nelson LM, Butwick AJ. Risk factors for prolonged postpartum length of stay following cesarean delivery. Am J Perinatol 2015;32(09):825-832

12 Nilsson IM, Kronborg H, Knight CH, Strandberg-Larsen K. Early discharge following birth - what characterises mothers and newborns? Sex Reprod Healthc 2017;11:60-68

13 Brown S, Small R, Faber B, Krastev A, Davis P. Early postnatal discharge from hospital for healthy mothers and term infants. Cochrane Database Syst Rev 2002;3(03):CD002958

14 Bayoumi YA, Bassiouny YA, Hassan AA, Gouda HM, Zaki SS, Abdelrazek AA. Is there a difference in the maternal and neonatal outcomes between patients discharged after $24 \mathrm{~h}$ versus $72 \mathrm{~h}$ following cesarean section? A prospective randomized observational study on 2998 patients. J Matern Fetal Neonatal Med 2016; 29(08):1339-1343

15 Liu S, Heaman M, Kramer MS, Demissie K, Wen SW, Marcoux S; Maternal Health Study Group of the Canadian Perinatal
Surveillance System. Length of hospital stay, obstetric conditions at childbirth, and maternal readmission: a population-based cohort study. Am J Obstet Gynecol 2002;187(03): 681-687

16 Clapp MA, Little SE, Zheng J, Kaimal AJ, Robinson JN. Hospital-level variation in postpartum readmissions. JAMA 2017;317(20): 2128-2129

17 Campbell OM, Cegolon L, Macleod D, Benova L. Length of stay after childbirth in 92 countries and associated factors in 30 low- and middle-income countries: compilation of reported data and a cross-sectional analysis from nationally representative surveys. PLoS Med 2016;13(03):e1001972

18 Gazmararian JA, Koplan JP. Length-of-stay after delivery: managed care versus fee-for-service. Health Aff (Millwood) 1996;15 (04):74-80

19 Krieger N, Chen JT, Waterman PD, Soobader MJ, Subramanian SV, Carson R. Geocoding and monitoring of US socioeconomic inequalities in mortality and cancer incidence: does the choice of area-based measure and geographic level matter? The Public Health Disparities Geocoding Project. Am J Epidemiol 2002;156 (05):471-482 